\title{
Selective inhibitors gain traction
}

A great deal of evidence points to the crucial role of mTOR in several human malignancies, perhaps reflecting its regulation by upstream oncogenic PI3K signalling pathways and its regulation of downstream cell growth, survival and proliferation pathways. mTOR can form two complexes, mTOR complex 1 (TORC1) and TORC2. However, the realization that TORC2 is insensitive to the allosteric mTOR inhibitor rapamycin, and that rapamycin increases PI3K activity by suppressing a negative feedback loop, together with recent reports that rapamycin is an incomplete inhibitor of TORC1, have led researchers to explore alternative mTOR inhibitors.

Reporting in Nature Medicine, David Fruman and colleagues compared rapamycin with the selective mTOR active site inhibitor PP242. Consistent with previous findings, inhibition of the mTOR active site blocked TORC1 and TORC2 signalling without aberrantly affecting

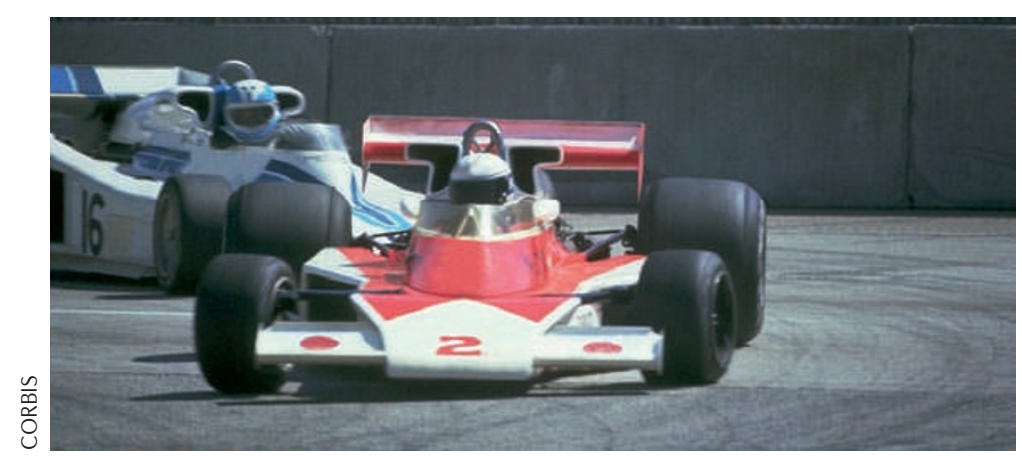

the negative feedback of PI3K in (Philadelphia chromosome positive $\left(\mathrm{Ph}^{+}\right)$) leukaemic cell lines. PP242 also had a greater cytotoxic activity and antiproliferative potency towards leukaemia cells than rapamycin. With a view to improving treatment outcomes, drug combinations were assayed in newly diagnosed and relapsed or refractory $\mathrm{Ph}^{+} \mathrm{B}$ precursor acute lymphoblastic leukaemia (B-ALL) patient samples. PP242, together with the second-generation tyrosine kinase inhibitor (TKI) dasatinib, exhibited significantly reduced colony formation compared with either drug alone or a combination of rapamycin and dasatinib.

Encouraged, the authors switched to in vivo models. In mice transplanted with p190 BCR-ABLexpressing mouse haematopoietic progenitor cells, PP242 dosing extended survival and enhanced the efficacy of the first-line BCR-ABL TKI imatinib more effectively than several mouse and human BCR-ABL rapamycin. However, $\mathrm{Ph}^{+}$patients with B-ALL can acquire resistance to this TKI. Therefore, on the basis of the earlier in vitro success with dasatinib, treatment with this drug was assessed in human $\mathrm{Ph}^{+} \mathrm{B}$-ALL cell lines and primary human $\mathrm{Ph}^{+}$ B-ALL xenograft models and found to synergize with PP242 resulting in disease regression. Together, these data suggest the potential therapeutic benefits of targeting both upstream tyrosine kinases and mTOR specifically through dasatinib-PP242 combination therapy.

The pan-PI3K-mTOR active site inhibitor PI-103 also exhibited strong anti- $\mathrm{Ph}^{+} \mathrm{B}-\mathrm{ALL}$ efficacy, so the authors sought to tease out the tolerability for rapamycin, PI-103 and PP242. As expected, rapamycin was immunosuppressive both in vitro and in vivo. But surprisingly, when administered alone and in combination with dasatinib, PP242 produced much less immunosuppression than PI-103.

Therefore, this promising blend of efficacy and tolerability in mouse models indicates that the further development of selective TORC1 and TORC2 inhibitors to treat leukaemia is warranted.

Kira Anthony, Editor, NCI-Nature Pathway Interaction Database

ORIGINAL RESEARCH PAPER Janes, M. R. et al. Effective and selective targeting of leukemia cells using a TORC1/2 kinase inhibitor. Nature Med. 13 Jan 2010 (doi:10.1038/nm.2091) 